\title{
Modelling The Temperature of Cometary Particles
}

\author{
Zhangfan Xing and Martha S. Hanner \\ Jet Propulsion Laboratory, 4800 Oak Grove Drive, Pasadena CA 91109
}

\begin{abstract}
We use the DDA method to study scattering properties of aggregates. Based on this aggregate model, the temperatures of cometary dust particles will be close to those of the equivalent total volume sphere when the aggregate porosity is $\leq 0.64$.
\end{abstract}

\section{Introduction}

Optical and thermal properties of grains are needed to interpret the observations of interplanetary and cometary dust, including the thermal emission from the cometary dust com $a$ and the zodiacal emission. Cometary dust particles are believed to be heterogeneous aggregates of grains a few tenths of a micron in size, based on examination of chondritic aggregate Interplanetary Dust Particles (IDPs) as described by Brownlee (1985).

While modelling has been done for fractal-type aggregates, the constituert grains, or "monomers", have usually been much smaller than the wavelength, essentially in the Rayleigh regime (Kozasa et al. 1992). West (1991) showed that the monomer size has a strong influence on the optical properties of the aggregate particle. Consequently. we have constructed aggregate particles as clusters of monomers which are similar in size to the grains in chondritic aggregate IDPs. To obtain their scattering properties, we have used the Discrete Dipole Approximation (DDA) method (Draine 1988, Draine and Flatau 1994) which represents the model particle as an array of radiating dipoles on a cubic lattice. One specific astrophysical problem re-examined here is the radiative equilibrium temperatures of the cometary dust exposed to the solar flux at several distances from the Sun.

\section{Modelling the Aggregates}

Shown in Figure 1 are six aggregates we have studied. Each aggregate consists of 10 monomers, either spheres or tetrahedra, of which one is positioned in the center and the other nine are located around the central one more or less randomly. There are three different kinds of aggregate structures, namely, "Overlapping", "Touching" and "Separated" The distance between the center of the surrounding monomers and that of the central one is 1, 2, and 3 times the monomer radius (or equivalent-volume radius for non-spherical monomer) respectively. When embedded in a cubic lattice as required by the DDA (Draine and Flatau 1994), individual monomers are represented by dipole arrays. We 


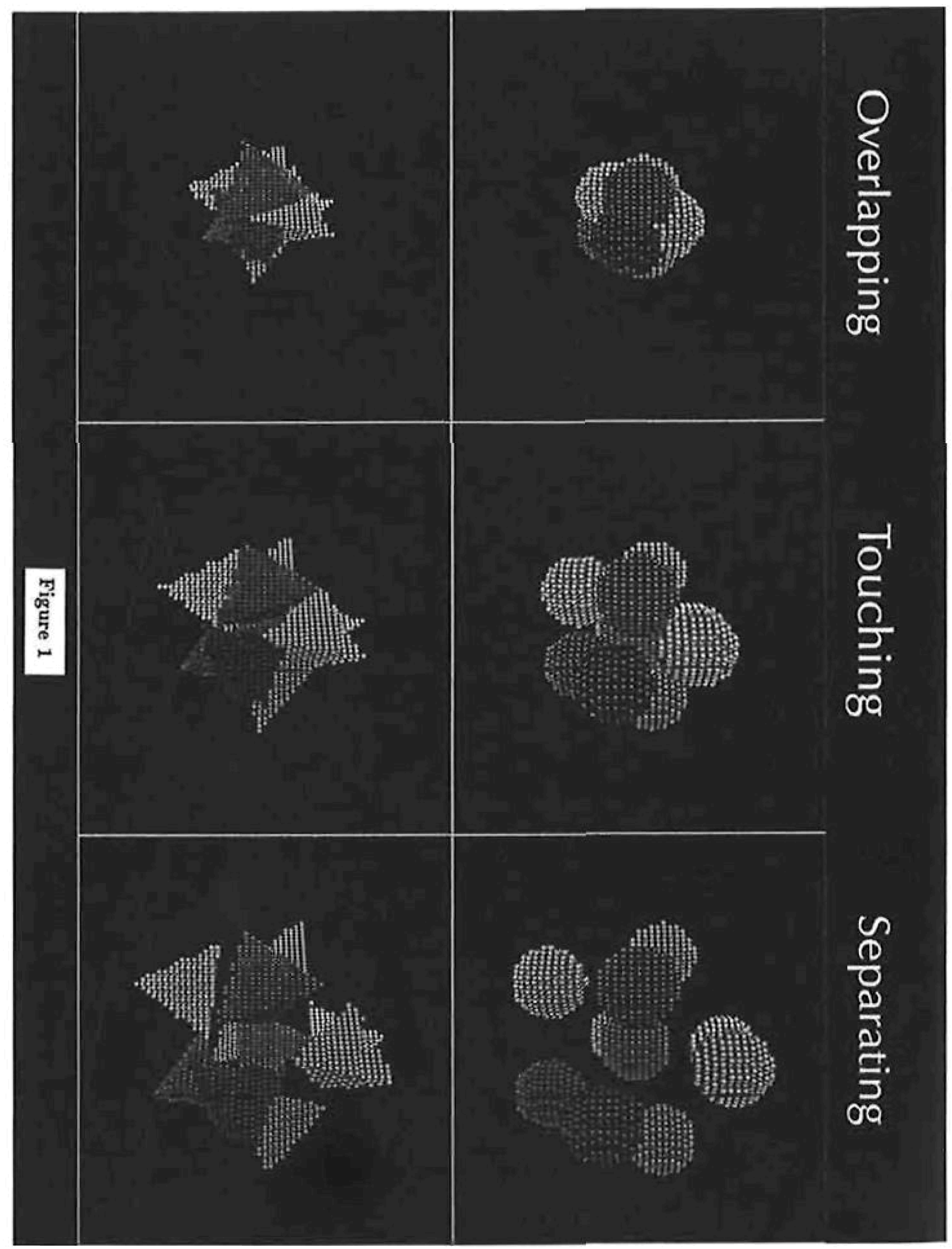


tried to use finer dipole arrays in order to make the study of shape effects valid. For aggregates in Figure 1, there are about 650 dipoles in each monomer. We denote $r_{m}$ as the equivalent volume radius of an individual monomer.

We will be primarily interested in the following questions:

First, how does the absorption cross section, $C_{a b s}$, depend on the morphology of the aggregates, e.g., monomer shape, relative positions of monomers, porosity of aggregates? Second, does $C_{a b s}$ deviate largely from that of a sphere or single tetrahedron of equivalent volume? Third, how does the temperature of an aggregate in the solar radiation field depend on the morphology?

We have carried out scattering calculations for $r_{m}=0.25 \mu \mathrm{m}$ and $0.5 \mu \mathrm{m}$. Both IDPs and comet dust are carbon-rich and low in albedo. Thus we used wavelength-dependent refractive indicies for glassy carbon from Edoh (1983). Presented in Figure 2 are the wavelength dependent values of the absorption cross section per volume, i.e., $C_{a b s} / V\left(\mu \mathrm{m}^{-1}\right)$, for aggregates in a single spatial orientation, assuming the light is coming from the bottom in Figure 1. The values for a single sphere and single tetrahedron with radius $r_{m}=0.25 \mu \mathrm{m}$ and $r_{m}=0.5 \mu \mathrm{m}$ are also plotted for comparison. The values for a single tetrahedron were obtained using the DDA method by averaging over $8 \times 9 \times 32$ spatial orientations. The tetrahedron was represented by 673 dipoles at $\lambda \geq 3 \mu \mathrm{m}$ and 10726 dipoles at $\lambda<3 \mu \mathrm{m}$. The values for a single sphere were however calculated by a standard Mie code. All computed values are represented as plus signs.

We further obtain the asymptotic $C_{a b s} / V$ value for each aggregate as well as single tetrahedron at $\lambda=0$, simply by counting the number of dipoles in the volume and the number of dipoles projected onto the plane perpendicular to the incident light, and taking into account the fact that $Q_{a b s}$ must be 1 at $\lambda=0$. These values are used to extrapolate the absorption values beyond $\lambda=1 \mu \mathrm{m}$.

Draine and Flatau (1994) stated that the validity range of the DDA method requires that the wave phase shift $|m| k_{0} d$ over a dipole cell be less than 1 . For aggregates shown in Figure 1 with $r_{m}=0.25 \mu \mathrm{m}$ or $r_{m}=0.5 \mu \mathrm{m}$, this criterion

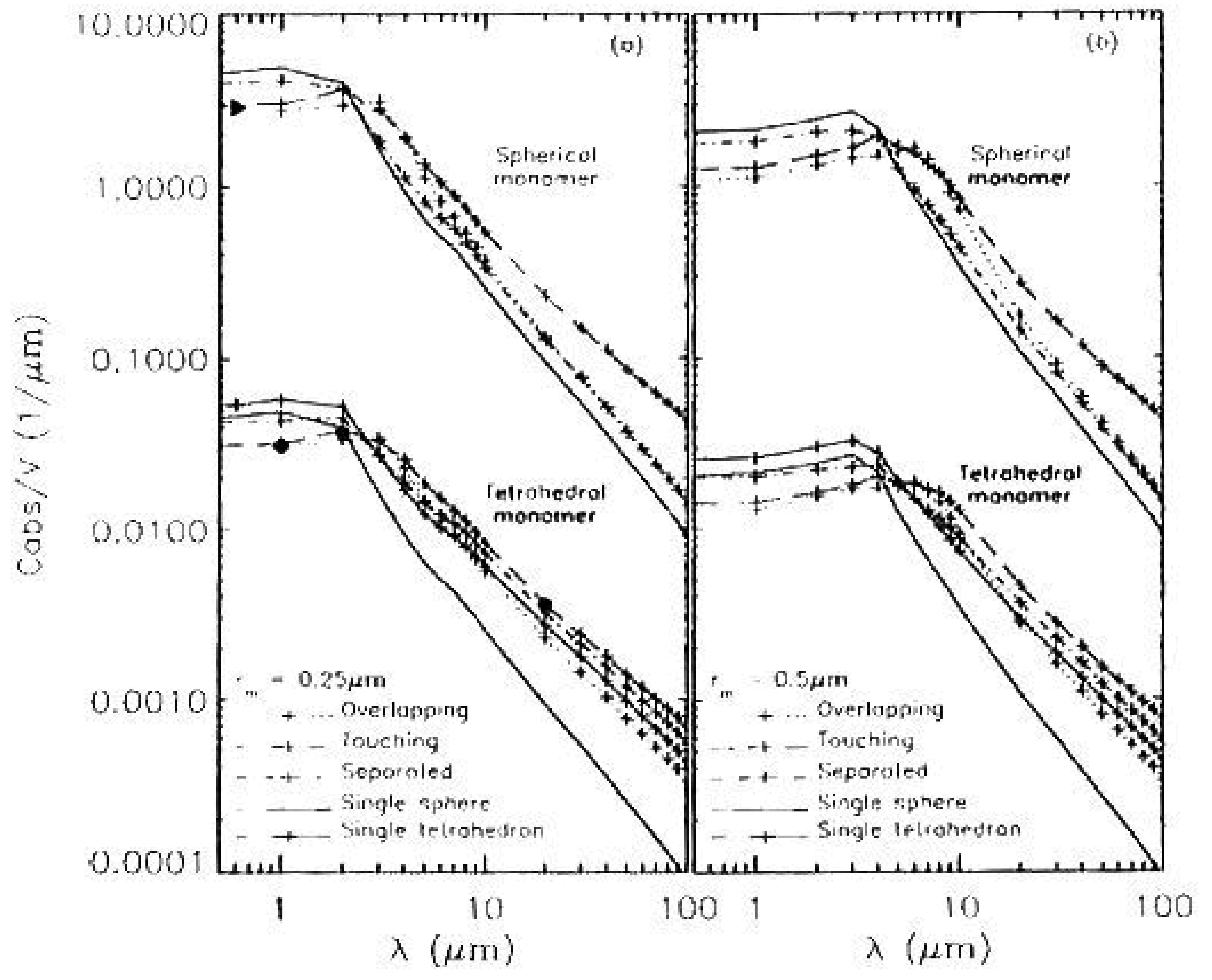

Figure 2 Absorption cross section per volume. (a) monomer size $r_{m}=0.25 \mu \mathrm{m}$ (b) $r_{m}=0.5 \mu m$. Tetrahedral cases are vertically shifted by a factor of $10^{-2}$. 
is satisfied at $\lambda>1 \mu m$ or $2 \mu m$ respectively. To assure that the absorption values at $\lambda \leq 2 \mu m$, including those by extrapolation, are accurate enough for our current discussion, we have made one more CPU-expensive calculation for the "Touching" aggregate of spherical monomers at $\lambda=0.6 \mu \mathrm{m}$ using about 5100 dipoles per monomer. In this case, $|m| k_{0} d=0.4934<1$. Its $C_{a b s} / V$ value is plotted as a triangle in Fig. 2(a), and the relative difference is smaller than $1 \%$ compared with the extrapolated value. This reflects the general insensitivity of absorption to light wavelength at $x_{m}=2 \pi r_{m} / \lambda<1$.

There are 3 filled circles in Fig. 2(a), which were calculated by averaging over $8 \times 9 \times 32$ orientations for the "Touching" aggregate consisting of tetrahedral monomers with $r_{m}=0.25 \mu m$ at $\lambda=1,2$ and $20 \mu m$. Their agreement with respective values for a single orientation confirms, our general experience with the DDA method that $C_{a b s}$ is not sensitive to the orientation for approximately equidimensional particles. Furthermore the geometric cross-section of our aggregates varies with orientation by $<2 \%$. Thus we have confidence in using absorption values for a single orientation to compute the temperature.

\section{The Temperature of Cometary Dust}

The equilibrium temperatures ${ }^{1}$ of cometary dust aggregates are calculated by using the following equation:

$$
\left(\frac{r_{0}}{r}\right)^{2}=\left(\int_{0}^{\infty} 4 \pi B(\lambda, T) C_{a b s}(\lambda) d \lambda\right) /\left(\int_{0}^{\infty} S(\lambda) C_{a b s}(\lambda) d \lambda\right)
$$

where $r_{0}=1 \mathrm{AU}, r$ in $\mathrm{AU}$ is the heliocentric distance of the dust grain, $C_{a b s}(\lambda)$ the absorption cross section of particles, $S(\lambda)$ the solar flux at $1 \mathrm{AU}$, and $B(\lambda, T)$ the Planck function for the emitting particle. The results are shown in Fig. 3 .

It is evident from Fig. 2 and 3 that aggregate structure, monomer shape, and size all influence the scattering properties. The temperatures in Fig. 3 can be understood by reference to $C_{a b s} / V$, specifically the ratio of $C_{a b s}$ at $\lambda<1 \mu \mathrm{m}$, where the solar flux is absorbed, to $C_{a b s}$ at $1-20 \mu \mathrm{m}$, where the absorbed energy is reradiated. Hanner (1983) showed that absorbing spheres $<1.5 \mu \mathrm{m}$ radius will be hotter than a blackbody, with temperature inversely correlated with size, and we see that $C_{a b s}$ decreases steeply in the infrared for single spheres. Since the slope of $C_{a b s}$ in the infrared is less steep for the aggregates, we expect that aggregates of submicron monomers will tend to be cooler than a single monomer.

When the monomers are touching (porosity, $P \approx 0.64$ ) or overlapping $(P \approx$ $0.3)$, the temperatures are the same as those of a sphere with volume equivalent to the aggregate, within $\sim 3 \%$. As the monomer separation increases, there is less interaction between them and the temperatures approach those of a single monomer. For the case of spherical monomers with $3 r_{m}$ separation $(P \approx 0.84)$, the temperatures are $4-6 \%$ lower than the monomer temperature. In contrast to the spheres. the separated tetrahedra have temperatures significantly lower than a spherical monomer and about $5-8 \%$ lower than that of a single tetrahedral monomer. Thús, the transition between temperatures close to the monomer and temperatures close to the total volume equivalent sphere occurs at porosities $>0.64$, regardless of monomer shape or size.

1 At heliocentric distances where sublimation is unimportant. 

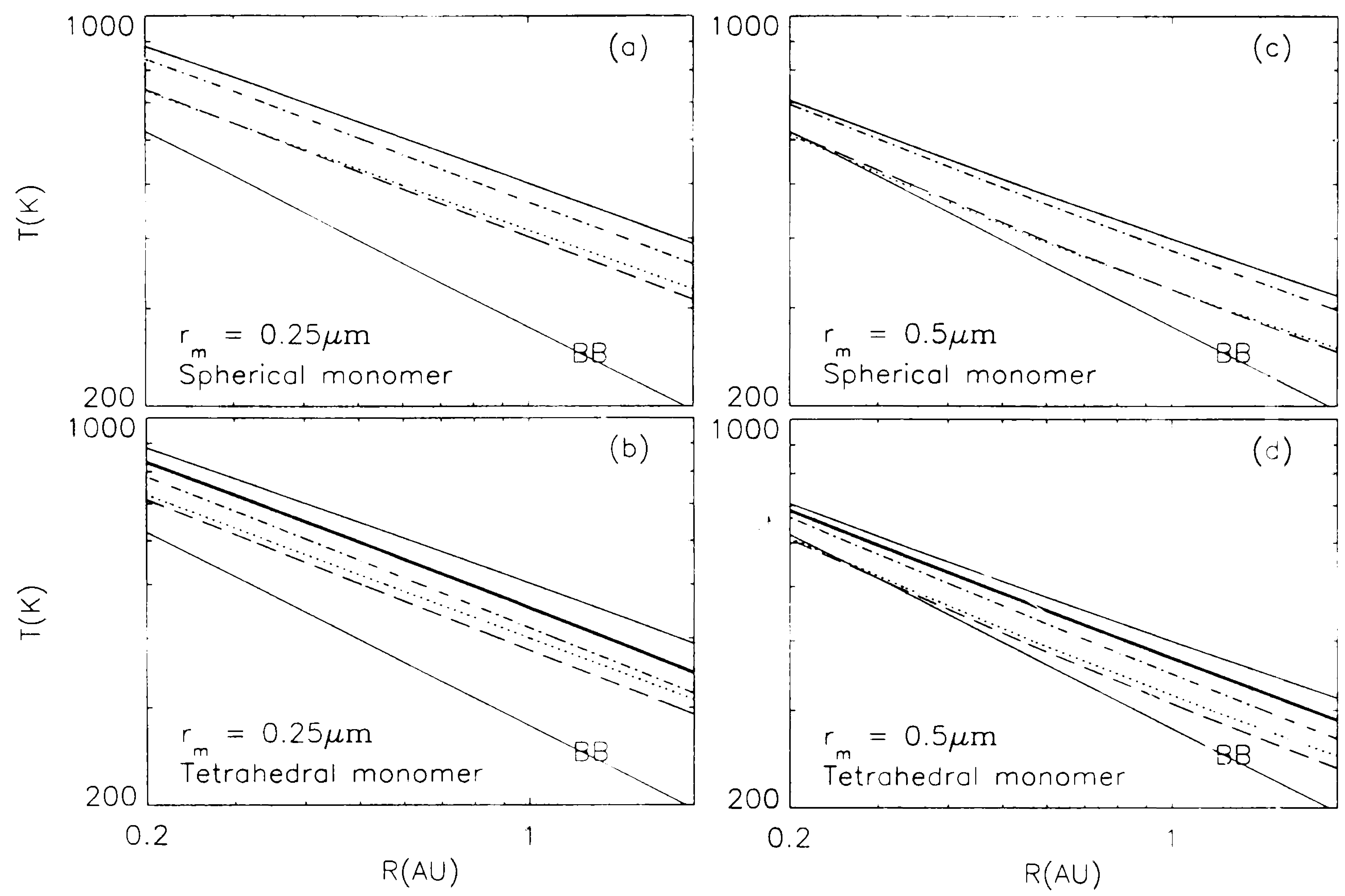

Figure 3. Temperazure versus heliocentric distance. single sphere; single tetrahedron; BB blackbody; … overlappng, --- touching, -.-. separated cases.

At small heliocentric distances, the Planck function peaks in the nearinfrared, on the flat part of the $C_{a b s} / V$ curve, and thus the temperatures approach the blackbody temperature.

We conclude that, if an aggregate consists of compact. equidimensional absorbing monomers a few tenths of a micron in size, the aggregate temperature will be close to that of an equivalent volume sphere when the porosity of the aggregate is $<0.64$. This aggregate model resembles the chondritic IDPs of likely cometary origin.

Acknowledgments. Research performed at the Jet Propulsion Laboratory, California Institute of Technology, under contract with NASA. Z. Xing was supported by a National Research Council Postdoctoral Fellowship

\section{References}

Brownlee, D.E. 1985, Ann. Rev. Earth Planet. Sci. 13, 147

Draine, B.T 1988, ApJ, 333, 848

Draine, B.T. and P.J.Flatau 199.1, J. Opt. Soc. Am. A 11, 1491

Edoh, O. 1983, Ph.D dissertation, Dept. of Phys.. Univ of Arizona

Hanner, M.S. 1983, in Cometary Exploration, ed. T.I. Gombosi, Vol. II, p.1

Kozasa $\mathrm{T}$ et al. 1992, A \& A 263, 423

West, R.A. 1991, Appl. Optics 30, 5316 